\title{
SWI, A New MRI Sequence - How Useful it is?
}

\author{
DR SHRINIVAS B. DESAI \\ Editor-in-Chief
}

Ind J Radiol Imag 2006 16:1:13-14

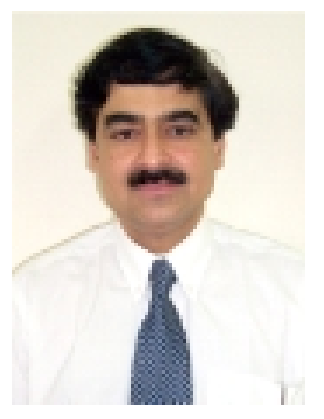

Susceptibility Weighted Imaging (SWI) is a new MR sequence, which evaluates and exploits the magnetic properties of blood, iron and other tissues. SWI is magnitude or phase images or combination of these two, obtained with $3 \mathrm{D}$ velocity compensated gradient echo sequence. As we know, paramagnetic substance like deoxyhemoglobin, a product of blood degradation, ferritin, a non-heme iron are known sources of magnetic susceptibility in the tissues. Iron accumulation has been known to be associated with Parkinson's disease, Huntington's disease and Alzheimer's disease. Deoxygenated venous blood causes magnetic field inhomogenity due to reduction of $\mathrm{T} 2^{*}$ and phase difference in vessel and surrounding tissue. Thus, deoxyhemoglobin behaves like contrast agent for visualisation of venous structures. In SWI, there is a kind of mixture of spin density, T1, T2*, CSF suppression and susceptibility sensitivity. SWI images reveal region of edema identical to FLAIR images because of short TR and comparatively longer TE, however, SWI does not reveal low signal in CSF because of low flip angle. Bartzokis $G$ et al have shown that contrast in MR is maximum when volume fraction is about 0.25 . The volume fraction required to attain maximum contrast depends on the product of the volume of the source times its susceptibility. This means that we can indirectly evaluate different components, either vessels or micro bleed, smaller than a voxel.

It is obvious that SWI images are best obtained at high field. SWI on 3T will be faster and will have higher SNR. SWI can be obtained on 1.5 and $1 T$ MR also. SWI is unique combination of high resolution $3 \mathrm{D}$ gradient echo, full flow compensation in all 3 directions, thin slices, filtering of phase images, high-lighting susceptibility by phase masking and better interpretation of data.

SWI has been shown to detect both the venous vasculature and hemorrhage within the brain tumors, not well appreciated on conventional MRI. Cha S et al have shown ample evidence of growth dependency of gliomas on angiogenesis of pathological vessels. Sehgal $\mathrm{V}$ et al have shown that SWI offers better information than T1 W1 contrast MRI in detecting extent, morphology, architecture, blood products and venous vessels in brain tumors. SWI can differentiate recurrent tumor from post surgical trauma. SWI has been found to be more sensitive than conventional gradient echo imaging for detecting suspected diffuse axonal injuries (DAI) in children. The attempt has been made to correlate lesions seen on SWI with Glasgow Coma Scale with positive results. SWI images allow number, size and location of the lesions well correlated with clinical status of the patient.

We know that T2 W1 MR images are sensitive to high flow vessels which reveal flow void, however, are less sensitive to small low flow vessels. TOF and phase contrast MRA are also good in evaluating high flow velocities, however, reveal decreased sensitivity and specificity when flow velocities are reduced. Hence, all these techniques are suboptimal in evaluation of malformed vessels, which essentially reveal multi directional flow and low velocity. SWI signal is not affected by low velocity venous blood flow and hence is highly sensitive for detecting venous malformations. One of the limitations of SWI is difficulty in differentiating small venous structure from micro bleed. However, pre and post contrast SWI images can confidently differentiate these two pathologies.

Majority of the lacunar infarcts are thought to be thrombotic manifestations of atheromatous disease and hence, can be well evaluated with SWI.

Ability of SWI in measuring non heme-iron in diseases like HD, PD, AD, MS, ALS, anemia, AIDS etc., may help in better understanding of the disease process and 

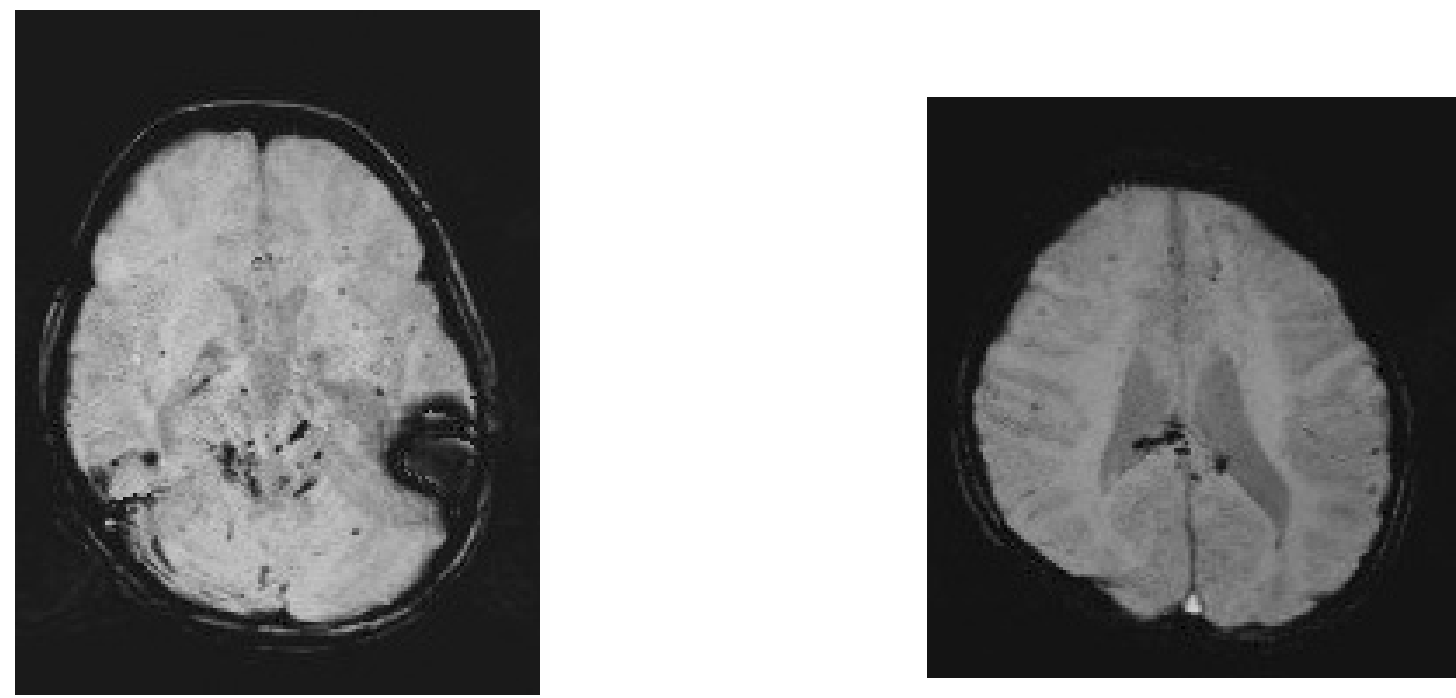

Fig 1a \& 1b: Multiple hypointense lesions are seen along the corpus callosum, bilatreal parietal white matter, crus cerebri, dorsal midbrain and pons and the basal gnaglia on SWI images

prediction of outcome.

SWI can be of great value in research and applications of Functional MRI as it can localize expected activation, to determine why activation occur in a particular brain region etc., This is best done at high field (3T, 4T) MRI.
We routinely use SWI for high-resolution venous anatomy, evaluation of tissue susceptibility and cerebro vascular diseases. 